\title{
I. Laute und Schriftzeichen (Buchstaben)
}

1 Wenn wir deutsch sprechen, benutzen wir folgende Laute:

1) Vokale oder Selbstlaute:

einfache Vokale : $a$ e $i$ o $u$

Umlaute : $\ddot{a} \quad \ddot{o} \ddot{u}$

Diphtonge

(Doppellaute) : au ei eu

2) Konsonanten oder Mitlaute:

$\begin{array}{lllllll}p & b & f & w & & m \\ t & d & \beta & s & r & l & n^{3} \\ k & g & c b^{1} s c h & j & & & \\ c b^{2} & b & & & n^{4}\end{array}$

${ }^{1}$ wie in: ich; ${ }^{2}$ wie in: ach; ${ }^{8}$ wie in: neun, Ende; ${ }^{4}$ wie in: lange, Enkel

Wenn wir deutsch schreiben, benutzen wir folgende Buchstaben:

$\mathrm{A}$ a, B b, C c, D d, E e, Ff, G g, H h, I i, J j, Kk, L l, M m, N n, O o, P p, Q q, R r, S s, T t, Uu, V v, Ww, Xx, Yy, Z z, Ä ä, Ö ö, Üü, B.

2 Vergleichen wir die Laute mit den Buchstaben, so stellen wir fest, daß nicht für jeden Laut ein Buchstabe vorhanden ist, aber auch nicht für jeden Buchstaben ein Laut.

Manche Buchstaben werden für verschiedene Laute gebraucht: ch $\quad c h$ (wie in: ich) ch (wie in: ach)

n $\quad n$ (wie in: lange)

$n$ (wie in: neun)

b $\quad b$ (wie in: lieben)

$p$ (wie in: liebt)

$\mathrm{d} \quad d$ (wie in: Hände)

$t$ (wie in: Hand)

Umgekehrt gebraucht man aber auch verschiedene Buchstaben für denselben Laut:

$\begin{array}{llll}\text { eu, äu } & e u & \mathrm{~s}, \mathrm{~B}, \mathrm{ss} & s \text { (stimmlos) } \\ \mathrm{ei}, \mathrm{ai} & e i & \mathrm{ks}, \mathrm{x} & k s \\ \mathrm{f}, \mathrm{v} & f & \mathrm{ts}, \mathrm{z} & t s \\ \mathrm{w}, \mathrm{v} & w & \mathrm{kw}, \mathrm{qu} & k w\end{array}$

Dazu kommt als weitere Schwierigkeit die Schreibung von Fremdwörtern, bei deren Aussprache fremde Lautbezeichnungen beibehalten werden:

$\begin{array}{llll}c & k, z & \text { th } & t \\ \text { ch } & k & \text { y } & \ddot{u} \\ \text { ph } & f & \text { eu } & \ddot{o}\end{array}$

Und schließlich können wir nicht hören, ob ein Wort mit einem großen oder mit einem kleinen Buchstaben beginnt. 